\title{
SUR LA RECEPTION DU MARXISME EN FRANCE : LE CAS ANDLER (1890-1920)
}

"... ce sont précisément les intelligences les plus distinguées et les plus solides du monde bourgeois qui se consacrent à une étude minutieuse de l'évolution du marxisme non pour l'anéantir, mais au contraire pour le comprendre. Dès lors il nous semble assez scabreux de vouloir démontrer ainsi le déclin du marxisme. "

Franz Mehring, Le Mouvement socialiste, 8 février 1902, p. 249.

On ne sait encore que peu de choses sur l'histoire du marxisme en France. Celle-ci ne se trouve qu'à l'état d'ébauches, de lambeaux d'études qui tranchent singulièrement avec la réputation d' " idéologie dominante » qu'eut le marxisme dans les années 1960 et 1970 . De cet âge d'or, rien n'est vraiment sorti. Depuis l'Histoire de Zévaès ${ }^{1}$ à celle de Dommanget ${ }^{2}$, sans oublier les pages importantes que Claude Willard consacra dans sa thèse à cette question ${ }^{3}$, les historiens s'étaient habitués ${ }^{4}$ à ne porter leur regard que vers un "marxisme orthodoxe ", le guesdisme, un marxisme réel au demeurant, dont le poids historique fut incontestable. Il ne nous revient pas de dire ici s'il était ou non conforme au texte. Quoi qu'il en soit, le guesdisme a longtemps incarné le marxisme à la face des hommes (il n'est qu'à rappeler la remarque que

1. Alexandre ZEvaks, De l'introduction du marxisme en France, Paris, Marcel Rivière, 1947.

2. Maurice Dommanget, L'Introduction du marxisme en France, Lausanne, Rencontre, 1969.

3. Claude Willard, Le Mouvement socialiste en France, les guesdistes (1893-1905), Paris, Editions sociales, 1965.

4. Cf. Thierry PAQUOT, Les Faiseurs de nuage. Essai sur la genèse des marxismes francais (1880-1914), Paris, Le Sycomore, 1980, qui note p. 11 : "C'est le marxisme du guesdisme qui constitue le principal objet de notre recherche, c'est dire combien celle-ci est limitée."

Revue de synthèse: IV S. No 1, janv.-mars 1989. 
fit Paul Leroy-Beaulieu après l'élection de Lafargue à la Chambre : "Avec M. Paul Lafargue, gendre de Karl Marx, ce qui entre dans le Parlement, c'est le collectivisme, c'est-à-dire une doctrine arrêtée, systématique. »). Il ne fut pourtant pas le seul. Les mérites conjugués, mais différents, de Madeleine Rebérioux ${ }^{5}$, d'une part, et de Daniel Lindenberg $^{6}$, d'autre part, ont été d'attirer l'attention sur des personnalités ou des courants marginaux du socialisme français. Tous deux ont contribué au décloisonnement de l'histoire intellectuelle du socialisme, la première en remettant en cause l'idée traditionnelle d'un clivage univoque entre un « jaurésisme " réformiste, humaniste, résolument non marxiste et... les autres, révolutionnaires, coupeurs de têtes, aveuglément marxistes; le second en faisant remarquer, à propos de Lucien Herr mais aussi de Georges Sorel ou de Charles Andler, que les bons connaisseurs de Marx, ceux qui ont tâché de le mieux comprendre et de le mieux faire connaître n'ont peut-être pas toujours été les marxistes les plus apparemment convaincus ${ }^{7}$.

Le cas Andler est assurément exemplaire. Né en 1866 à Strasbourg, Andler est surtout connu pour son ceuvre de germaniste. Maître de conférence à l'École normale supérieure dont il avait été l'élève, professeur à la Sorbonne puis au Collège de France, Andler fut aussi un socialiste de grande envergure en dépit des relations orageuses qu'il eut avec le Parti socialiste. La polémique qui l'opposa à Jaurès en 1913 fut un événement remarqué, mais il ne rompit tout à fait avec la S.F.I.O. qu'au sortir de la Première Guerre mondiale. Spécialiste de Nietzsche dès

5. Cf. plus particulièrement sa contribution à l'Histoire générale du socialisme, Jacques DROz, dir., "Le socialisme français de 1871 à 1914 ", p. 133-236 et « Jean-Jaurès et le marxisme", in Histoire du marxisme contemporain, sous la dir. de Dominique GRISONI, Paris, U.G.E., 1977, t. III, p. 27-43. Il faut ajouter les travaux de Georges HAUPT sur l'Internationale, en particulier : "De Marx au marxisme", Forschungen zur Geschichte Osteuropas, 20, 1978, p. 108-120, republié in G. HAUPT, L'Historien et le mouvement social, Paris, Maspero, p. 77-107.

6. Cf. Daniel LINDENBERG, Le Marxisme introuvable, Paris, Calmann-Lévy, 1975; ID., "Entretien avec H. Cortières et R. Pierre ", Communisme, 31-32, 1978, p. 18; ID., " Herr, Andler, Sorel. Trois intellectuels décomposent le marxisme ", in Michel CharZAT, dir., Georges Sorel, Cahier de l'Herne, 53, 1986, p. 192-201.

7. Michelle Perrot fait remarquer: "Le guesdisme [...] ne doit pas être considéré comme la voie royale, ni même peut-être comme la route principale de sa pénétration », Annales E.S.C., mai-juin 1967, « Les guesdistes : controverse sur l'introduction du maxxisme en France", p. 709. Cf. Michele MAGGI, La Formazione dell'egemonia in Francia. L'ideologia nella Terza Republica tra Sorel e Durkheim, Bari, De Donato, 1977, qui consacre tout un développement à Andler (p. 231-248), et en qui l'auteur voit, malgré son socialisme non marxiste, un des principaux médiateurs du marxisme en France aux côtés de Lafargue et de Deville. Il faut noter qu'un auteur aussi résolument non socialiste que l'était Elie Halévy était dès les années 1890 un excellent connaisseur de l'œuvre de Marx. II anima la discussion qui suivit l'exposé sur le matérialisme historique que fit Sorel en 1902 à la Société française de philosophie (cf. les deux lettres inédites de Sorel à E. Halévy du 24 mars et du 7 avril 1902 gracieusement communiquées par $M^{\text {me }}$ Guy-Loe). 
le début du siècle, il consacra au philosophe une cuvre gigantesque qui fit oublier ses travaux d'avant-guerre dans lesquels il discuta la doctrine de Marx $^{8}$.

L'histoire du marxisme se trouve donc à l'intersection de deux histoires, celle des intellectuels et celle du socialisme. Et l'on ne s'étonnera pas d'y trouver souvent comme principaux protagonistes, des marginaux, intellectuels situés aux lisières du monde académique et du monde militant. Personnages d'ailleurs souvent déroutants, aux démarches intellectuelles complexes: Andler n'est pas sans rappeler un Sorel ou un Péguy 9 .

On ne comprendrait rien, en outre, à la complexité des rapports qu'Andler entretint avec l'œuvre de Marx si l'on omettait d'évoquer ceux que ce grand germaniste français eut plus généralement avec la culture allemande. Nous y reviendrons. Mais ce trait vaut d'être appuyé, tant il paraît dépasser le seul cas d'Andler. La question nationale est sans doute l'une de celles qui, en France, anime souterrainement le débat intellectuel de la fin du siècle. Elle en vient troubler les enjeux initiaux. Andler était issu d'une famille alsacienne ayant tardivement opté (1879). L'attachement national vient compliquer davantage ses rapports avec le marxisme. Les trois périodes de l'interprétation andlérienne du marxisme l'ont chacune pour clé.

Ces trois temps ne constituent donc qu'une grille de lecture non sans faille. Ils permettent simplement de récuser l'idée reçue qui fait de Charles Andler l'un des initiateurs d'un socialisme français antimarxiste et, d'une certaine façon, l'un des pères d'un Henri de Man. Qu'il ait été intéressé par les idées d'Au-delà $d u$ marxisme ne fait aucun

8. Pour les éléments biographiques, on pourra se reporter à Ernest TonNeLAT, Charles Andler, sa vie et son aeuvre, Strasbourg, Publications de la Faculté des lettres de Strasbourg, 1937, qui comprend une bibliographie partielle de l'œuvre d'Andler. On pourra également consulter la notice établie par Justinien RAYMOND dans le Dictionnaire biographique du mouvement ouvrier dirigé par Jean MAIrRON, Paris, Éd. ouvrières, 1972. Remarquons d'emblée qu'Andler n'a jamais écrit de livre sur le marxisme. Sa critique est éparpillée dans plusieurs préfaces, articles ou commentaires. Il fut en revanche l'auteur d'ouvrages fort importants comme Le Prince de Bismarck, Paris, G. Bellais, 1899 et surtout de quatre volumes sur Nietzsche publiés de 1921 à 1931. Il faut, enfin, signaler que l'on trouvera des informations précieuses concernant l'élaboration du travail d'Andler dans les lettres de Sorel à Croce publiées par La Critica, dans ses volumes XXV à XXVIII. Sur la partie nietzschéenne de l'œuvre de Charles Andler, cf. Donato LONGO, La Présence de Nietzsche dans les débats politiques et culturels en France pendant l'entre-deux-guerres, 1919-1940, thèse de $3^{\mathrm{e}}$ cycle, Paris VIII, 1985.

9. Sur l'exemplaire de La Civilisation socialiste (Centre Charles Péguy, Oriéans) qu'Andler offrit à Péguy, on peut lire: "Charles Péguy, en souvenir de quelques idées communes, restées les mêmes. " Andler avait, en vain, essayé de réconcilier Herr et Péguy : "J'ai essayé autrefois, à votre insu, de plaider auprès de lui votre cause, d'amener un rapprochement " (lettre de Charles Andler à Charles Péguy, 3 septembre 1913, Centre Charles Péguy, Orléans). 
doute $^{10}$. Qu'il ait inauguré une tradition méta-marxiste, allant de la fin du $\mathrm{XIX}^{e}$ siècle à l'entre-deux-guerres, est beaucoup plus douteux. Andler avait surtout l'âme d'un professeur et l'esprit d'un grand érudit. Ses propres ambitions théoriques (s'il en eut) ont été reléguées par son travail critique. Il fut en cela, comme l'écrit Michele Maggi, un héritier direct du positivisme.

CHARLES ANDLER ET LE MARXISME : RENCONTRE ET CRISE (1890-1903) 11

Andler bénéficiait d'un avantage certain sur bon nombre d'intellectuels français : il connaissait parfaitement la langue allemande. Immense avantage lorsque l'on envisage le retard avec lequel l'œuvre de Marx était traduite en France... Sorel qui, ignorant l'allemand, dut se fier aux traductions disponibles ou aux bons offices d'Andler lui-même, en sut quelque chose ${ }^{12}$. En traduisant le Manifeste communiste (1901), Andler contribuait donc aussi à rendre plus accessibles des textes difficiles à trouver. Laura Lafargue avait fourni en 1895 une nouvelle édition de la traduction du Manifeste qu'elle avait déjà faite pour l'Égalité en $1882^{13}$ : c'était alors la plus récente.

La familiarité d'Andler avec la langue allemande lui avait permis d'acquérir une vaste culture de germaniste. Sur les registres des prêts de l'École normale supérieure, Hegel côtoie Schopenhauer (dès 1884), Effertz (en novembre 1896, Andler, alors " archicube », emprunte Arbeit und Baden), Rodbertus, Dühring, Menger, Lassalle mais aussi Max Weber (en octobre 1897, il lit la Römische Agrargeschichte). Sur les 300 livres environ qu'il emprunta durant sa scolarité à l'École, la moitié est composée d'ouvrages d'auteurs allemands. L'analyse de sa bibliothèque donnée à l'École normale supérieure (vraisemblablement incomplète) confirme cette ouverture sur la culture allemande moderne. A l'appui de notre propos, on y peut relever plusieurs ouvrages d'Eduard

10. L'ouvrage figure dans la bibliothèque Andler de l'E.N.S. Ulm.

11. Cf. Shlomo SAND, Georges Sorel et le marxisme. Rencontre et crise (1893-1902), thèse de $3^{e}$ cycle, Paris VII, 1982.

12. Sur la chronologie des ceuvres de Marx traduites en français, cf. T. PAQuot, op. cit. supra n. 4, p. 71-74. Sur les rapports Andler-Sorel, nous renvoyons une fois pour toutes à S. SAND, L'Illusion du politique. Georges Sorel et le débat intellectuel 1900, Paris, La Découverte, 1985, en part. p. 139-141. Sur ce sujet, cf. aussi Daniel LINDENBERG, " Herr, Andler, Sorel... ", art. cit. supra n. 6.

13. Bert ANDREAs, Le Manifeste communiste de Marx et Engels. Histoire et bibliographie, 1848-1918, Milan, Feltrinelli, 1963. 
Bernstein ${ }^{14}$ mais aussi toute une série d'ouvrages écrits par ceux qui, aux yeux d'Andler, prenaient part à la "décomposition du marxisme", Benedetto Croce ${ }^{15}$, Christian Cornelissen ${ }^{16}$, Giovanni Gentile ${ }^{17}$, Alfred Nossig ${ }^{18}$, Georges Sorel ${ }^{19}$. La bibliothèque comprend aussi le Capital et la Critique de l'économie politique dans leur langue originale ainsi que plusieurs ouvrages sur Marx qui attestent, malgré les préoccupations de plus en plus nietzschéennes d'Andler ${ }^{20}$, le constant intérêt qu'il porta à l'œuvre de Marx jusqu'à la Première Guerre mondiale au moins.

En 1891, Andler s'était rendu à Londres. Il y avait rencontré Engels à qui il s'était présenté comme "un débutant dans la littérature et dans le socialisme $" 21$. Cette visite avait pour but d'enrichir son information en vue d'un livre sur les origines philosophiques du socialisme allemand. Il y prévoyait un chapitre sur Marx. Cet ouvrage ne verra pas le jour mais de ce séjour, Andler rapporta sans doute une forte impression qui imprégna par la suite toute sa vision du socialisme allemand et du " marxisme orthodoxe ". Il avait lu les deux premiers volumes du Capital dès 1889 mais, de son propre aveu, il se sentait alors plus proche des anarchistes qu'il fréquentait par l'intermédiaire de son ami BernardLazare, que des marxistes. Au cours de son voyage à Londres, il avait également rencontré Sidney Webb, Béatrice Potter et Graham Wallas qui avaient suscité chez lui " la plus vive admiration "22. Son adhésion, enfin, au parti possibiliste de Paul Brousse, puis son ralliement au P.O.S.R. de Jean Allemane confirment la méfiance qu'il éprouvait à l'encontre du

14. En part., Zur Geschichte und Theorie des Sozialismus, Berlin, 1901; Wie ist wissenschaflicher Sozialismus möglich?, Berlin, Verlag der sozialistischen Monatshefte, 1901; Die Voraussetzungen des Sozialismus und die Aufgaben der Sozialdemokratie, Stuttgart, Verlag von Y. W. Dietz Nachf, 1899 ; Zur Frage : Sozialliberalismus oder Collectivismus?, Berlin, Verlag der sozialistischen Monatshefte, 1900.

15. En part., Recenti interpretazioni della teoria marxistica del valore. E polemiche interno ad esse, Turin, 1899 ; Per la interpretazione e la critica di alcuni concetti del marxismo. Memoria letta all' academia pontaniana nella tornata del 21 novembre 1897, Naples, 1897 ; Le Teorie storiche del prof. Loria, Naples, 1897 (article publié en 1896 dans le Devenir social). Croce avait connaissance des travaux d'Andler.

16. Théorie du salaire et du travail salarié, Paris, Giard et Brière, 1908; Théorie de la valeur. Avec une réfutation des théories de Rodbertus, Karl Marx, Stanley Jevons et BoehmBanerk, Paris, Giard et Brière, 1902.

17. La Filosofia di Marx, Pisa, Enrico Spoerri, 1899.

18. Revision des Sozialismus, Berlin/Bern, 1901.

19. Le Système historique de Renan, Paris, Jacques, s.d. Avec une dédicace : “A M. Andler. Souvenir très cordial. "

20. Cf. E. TonNelat, op. cit. supra n. 8.

21. Lettre de Charles Andler à Engels du 20 juin 1891, citee in ibid., p. 43.

22. Charles AndLer, Vie de Lucien Herr (1864-1926), Paris, Rieder, 1932, réed., Paris, Maspero, 1977, p. 118. 
marxisme français. La "scandaleuse insuffisance scientifique d'un Paul Lafargue ${ }^{23}$ l'indignait.

On ne sera guère surpris, dans ces conditions, du ton polémique que prirent ses premiers écrits sur Marx. Chargé par Luc Gersal, l'auteur d'un livre sur Berlin ${ }^{24}$, de rédiger une note sur le socialisme berlinois, Andler (qui écrivait alors sous le pseudonyme de Théodore Randal) se livra à quelques attaques incisives contre la social-démocratie allemande et, audelà, contre sa doctrine : le marxisme. Cette critique à double détente, habilement confuse parfois, restera le trait dominant de la lecture andlérienne du marxisme. La domination sans partage du « marxisme orthodoxe " sur le socialisme allemand - « Marx est pour eux aussi infaillible que le pape, il a même un successeur, Frédéric Engels, son ami, qui est aussi le dépositaire de ses papiers et de ses secrets ${ }^{25}$ - en était la partie faible.

Andler n'aura de cesse de dénoncer le caractère ecclésial du marxisme allemand, dogme figé que l'on n'enrichissait plus de nouvelles études et qui n'attendait plus que la réalisation de la prédiction d'Engels : « Entre deux bouffées de sa pipe, le célèbre socialiste anglais a prédit pour 1898 l'écroulement de la société actuelle ${ }^{26}$. Cette critique externe du marxisme visait encore plus son rôle historique, sa dégradation en quelque sorte, que la doctrine elle-même. Andler ne se prononçait pas encore sur le contenu de la pensée de Marx. Il lui rendait même hommage, non sans quelque ironie certes, mais celle-ci dirigée surtout contre les glossateurs allemands :

« En un sens, le Kapital de K. Marx (paru en 1867) a été fatal au socialisme allemand par sa grandeur même. En dehors de la pensée marxiste, personne n'ose plus penser, on n'ose même plus interpréter cette pensée du maître. On s'en va chez F. Engels s'informer timidement des passages obscurs. Engels a été le confident de la pensée du maitre, comme il est le dépositaire de ses manuscrits. Marx n'a rien dit sur la société future, donc on n'en dira rien, on aurait peur si l'on pensait par soi-même, d'endommager la pensée du maitre ${ }^{27}$.

Ce gel intellectuel que générait le marxisme n'était pas propre au socialisme allemand. Andler avait en tête, d'évidence, l'exemple du marxisme français, sur lequel régnait la « Sainte famille ». Son ironie ne

23. Ibid.

24. Luc Gersal, L'Athènes de la Sprée, Paris, Albert Savine, 1892.

25. Ibid., "Notes sur le socialisme berlinois" (rédigées par C. ANDLER), p. 234.

26. Ibid., p. 246 , n. 1.

27. Ibid., p. 245-246. 
s'atténuera guère : le mode affectif sur lequel un patrimoine intellectuel était géré étonnera toujours son esprit positif. Dans une lettre à Marie Allart, la secrétaire de l'École socialiste qu'Andler contribua à lancer à l'automne 1909, il écrit :

" Le petit Longuet considère Marx comme un Capital qu'il faut se transmettre, et que les descendants de Marx ont à gérer [...]. Dites-lui, selon sa formule, que les “démocrates" qui parlent de "décomposition du marxisme" ne l'emportent pas encore, et que nous vénérons toujours son grand-père ${ }^{28}$.

L'incarnation historique du marxisme ne fut pas le seul objet des études andlériennes. Y prit place aussi un autre type de critique, plus fondamental. Tout en lisant Marx avec attention et précision, Andler n'a jamais réalisé ce "retour à Marx " que Sorel espérait en ces mêmes années. Andler fit toujours sa lecture en historien et en érudit. Sorel accompagna la sienne d'un travail théorique et d'une philosophie. La "décomposition du marxisme" a un sens positif et philosophique chez Sorel, elle en revêt un, négatif et politique, chez Andler.

A partir de 1895 et jusque vers 1902-1903, Andler se livra à une offensive critique contre les bases théoriques du marxisme. C'est une période où fleurissaient en France les premières revues marxistes, l'Ėre nouvelle, le Devenir social, le Mouvement socialiste dans lesquelles Andler n'écrivit point. Leur caractère militant le rebutait. C'est une époque aussi où le marxisme, selon l'expression consacrée par Masaryk, connaît sa première crise dans plusieurs pays européens.

La publication du troisième volume du Capital permit à Andler d'en faire un compte rendu dans la Revue blanche ${ }^{29}$. Il ne s'agissait plus là des marxistes mais de Marx lui-même : œuvre immense, certes, mais fausse. Le baroque du vocabulaire employé par Andler dans cette critique occulte l'attaque frontale. Celle-ci est pourtant bien réelle. Il reproche à l'œuvre majeure de Marx sa faiblesse : celle-ci n'a que les apparences de la force et de la solidité : "Les morceaux du Capital sont d'un grain excellent et solide. L'ensemble se disjoint ${ }^{30}$. Plus précisément, Andler accusa Marx d'avoir négligé la part du bénéfice commercial et celle de la rente foncière. Dans ce court compte rendu, l'essentiel des reproches

28. Lettre de C. Andler à Marie Allart, s.d. (automne 1911 ou 1912), Paris, Institut français d'histoire sociale (I.F.H.S.), 14 AS 188 (fonds Andler).

29. C. ANDLER, "La fin du "Capital" de Marx ", La Revue blanche, 15 mai 1895, p. $450-454$.

30. Ibid., p. 454 . 
qu'Andler ne cessera de faire à Marx, des années durant, est en place. L'œuvre de décomposition a commencé.

Ce fut l'année suivante, en 1896, qu'Andler débuta un cours sur Marx, au Collège libre des sciences sociales ${ }^{31}$. Andler eut longtemps le projet de le publier. Plusieurs lettres adressées à Georges Renard ${ }^{32}$ montrent que ce projet fut sur le point d'aboutir sans que l'on sache pourquoi il ne vit jamais le jour. Andler ne fit à ce sujet qu'une remarque, emplie de mystère : "Je n'ai pas à m'exprimer sur les circonstances qui ont empêché la publication de ces leçons ${ }^{33}$. Sorel sut mettre à profit le titre de ce cours qui s'étendit sur deux années en publiant la Décomposition du marxisme. De ce passage au Collège libre des sciences sociales, où Andler fut d'ailleurs plus qu'un simple professeur ${ }^{34}$, nous n'avons comme traces que quelques articles dispersés qui donnent le ton de cette série de conférences.

Dans le même temps qu'il enseignait au Collège libre, Andler achevait une thèse soutenue en 1897. Elle portait sur les origines du socialisme d'État en Allemagne ${ }^{35}$. Marx y est peu présent. Il n'y est pas fait référence avant la page 182 si ce n'est pour remarquer, dès les premières lignes, « que Karl Marx a causé la stérilité de toute la littérature révolutionnaire " et qu'en ce sens, il se rapproche de Rodbertus dont le système " arrêta les recherches des socialistes d'État » ${ }^{36}$.

Andler établit un parallèle plus profond entre les deux penseurs. S'il fait justice à Marx de l'accusation portée contre lui par Rodbertus, qui s'était cru pillé sans être nommé, il découvre chez l'un et l'autre de fortes similitudes de pensée. Ces rapprochements sont d'ailleurs pour Andler des angles d'attaque : interprétation, fausse selon lui, de la rente foncière comme une participation au profit social, incompréhension du bénéfice commercial et surtout erreur sur la valeur-travail :

31. Sur ce Collège fondé par Dick May, cf. Christophe Prochasson, « Sur l'environnement intellectuel de Georges Sorel : l'Ecole des hautes études sociales (1899-1911)», Cahiens Georges Sorel, III, 1985, p. 16-38.

32. Lettre de Charles Andler à Georges Renard du 2 août 1897, Fonds Georges Renard, t. 96, MS 2577, Paris, Bibliothèque historique de la ville de Paris (B.H.V.P.) : « $M a$ Décomposition du Marxisme (où vous figurez) ne sera pas prête avant l'hiver. Le néomarxisme juridique n'en est qu'un chapitre. "

33. C. ANDLER, « Un système nouveau de socialisme scientifique : M. Otto Effertz », Revue de métaphysique et de morale, 1906, p. 597.

34. Plusieurs lettres de Dick May à Georges Renard, Fonds Georges Renard, t. 131, MS 2612, Paris, B.H.V.P. (en part. celles du 11 oct. 1895 et du 7 déc. 1896) montrent qu'Andler a joué un rôle important dans les débuts du Collège.

35. C. ANDLER, Les Origines du socialisme d'Etat en Allemagne, Paris, Alcan, 1897.

36. Ibid., p. 3 . 
" Ils disent que des industries diverses sont inégalement productives pour un même travail. Mais mesurant toutes choses par le travail, ils n'ont pas le droit de dire cela, et le même travail doit engendrer nécessairement, dans leur théorie, des valeurs égales. Pourtant ils constatent avec raison que le produit d'un travail également grand en diverses industries ne serait pas échangeable contre une somme égale des produits de toutes les autres industries. Il ne vaut pas de même. Et voilà leur dogme en ruines devant le simple fait de cette différence. Mais ce qui peut faire cette différence, puisque ce n'est pas le travail, c'est uniquement que des produits divers sont inégalement désirés $[\ldots]$ ) ${ }^{37}$.

Peu à peu, Andler précisait ses arguments. Son objectif est bien alors la ruine des fondements du marxisme qu'il envisage d'abord comme un objet d'érudition. Ses deux années de cours au Collège libre des sciences sociales (1896-1897) lui ont permis d'approfondir sa critique. Il recherche des appuis théoriques chez tous ceux qui « revisent » Marx ou « reviennent " à lui. Sorel est un de ceux-là bien que leurs rapports n'aient pas toujours été marqués par l'aménité et aient vraisemblablement été cassés par le rapprochement des "soréliens » de l'Action française : «Où se terrent les petits "Sorellistes"? Edouard Berth? Pourquoi ne parlent-ils pas? Ils n'ont pas tous passé à l'Action Française ? " ${ }^{38}$ D'autres auteurs attirèrent alors l'attention d'Andler, même lorsque leurs systèmes sont "faux ", comme Croce ${ }^{39}$ ou Labriola ${ }^{40}$. En revanche, il trouve d'immenses qualités à L'Utilité sociale de la propriété individuelle d'Adolphe Landry ${ }^{41}$, disciple d'un auteur auquel Andler se référa très souvent avec enthousiasme, Otto Effertz, un « socialiste allemand d'une espèce hétérodoxe ", qu'il fit inviter par Dick May à l'École des hautes études sociales $^{42}$. Le Viennois Anton Menger, professeur de droit, frère de l'économiste Carl Menger qui fonda l'école marginaliste, fut, lui aussi, étudiè...

Tous ces auteurs permirent à Andler de nourrir sa critique. De Labriola, il retint le refus de la « métaphysique de Marx », rejetant toute

37. Ibid., p. 418.

38. Lettre de Charles Andler à Marie Allart du 13 mai 1910, Paris, I.F.H.S. 14 AS 188.

39. Compte rendu (anonyme) du livre de Benedetto CROCE, Materialismo storico ed Economia marxistica, "Saggi critici", Milano/Palermo, Sandron, 1900, dans Notes critiques-Sciences sociales, 4, 25 févr. 1900, p. 77-78.

40. C. ANDLER, "La conception matérialiste de l'histoire d'après M. Antonio Labriola", Revue de métaphysique et de morale, nov. 1897, p. 644-658.

41. Compte rendu dans Notes critiques-Sciences sociales, 7, 25 juil. 1901, p. 218.

42. Lettre de Charles Andler à Marie Allart du 12 février 1912, Paris, I.F.H.S., 14 AS 188. 
détermination, tout mécanisme. Il puisa chez Sorel ${ }^{43}$ des remarques sur l'insuffisance des explications faites par Marx des relations infrastructure /superstructure et récusa l'idée que lorsque « l'infrastructure est changée la superstructure s'effondre ${ }^{44}$ :

« C'est pourquoi, avec $\mathrm{M}$. Sorel, nous préférerions voir une contingence non seulement dans la genèse primitive des modes de produire, mais dans toutes les médiations qui existent entre cette infrastructure prétendue et les produits supérieurs. Seulement, de celui qui pense ainsi, nous dirons, à son éloge, ce que M. Sorel, avec sévérité, disait d'un autre : qu' "il rejette tout, absolument tout, de la doctrine de Marx" $"{ }^{45}$.

Si Sorel et Labriola servaient nommément à livrer bataille contre le déterminisme, Menger fut un appui pour dévoiler la faiblesse des fondements juridiques du marxisme. La mise en avant du Droit n'est pas non plus une démarche étrangère à Sorel ${ }^{46}$. Andler sut d'ailleurs renvoyer aux Saggi di Critica del Marxismo : " L'esprit juridique est de nature, comme l'a dit Georges Sorel, à diminuer efficacement la croyance ouvrière au pouvoir magique de la force $"{ }^{47}$. Mais ce fut Menger et non Sorel qu'Andler préfaça. Tout en reprochant à cet auteur d'avoir " gardé de l'économie marxiste ", " ses fondements les plus contestables " ${ }^{48}$, il lui savait gré d'avoir tenté de répondre au «mépris " que Marx avait pour le droit. Car « tout le problème de la justice sociale » était, selon Andler, qu'il avait « une face économique et une face juridique " ${ }^{49}$. Le mouvement ouvrier élaborait un droit nouveau :

" Dans les profondeurs du peuple s'élaborent depuis un siècle les principes de $\propto$ droit nouveau. Ce droit naissant, le code en est ébauché dans les systèmes socialistes, et le mouvement socialiste n'est qu'un effort continu pour le faire aboutir $[\ldots]{ }^{50}$.

43. Cf. S. SAND, op. cit. supra n. 12, p. 74-97.

44. C. ANDLER, art. cit. supra n. 40, p. 655.

45. Ibid., p. 657-658.

46. Cf. S. SAND, "Lutte de classes et conscience juridique dans la pensée de Georges Sorel ", in Jacques Julliard, Shlomo SAND, Georges Sorel en son temps, Paris, Seuil, 1985, p. 225-245, et Patrice Rolland, «L'enjeu du droit ", in M. Charzat, dir., op. cit. supra n. 6, p. 28-44.

47. Préf. de C. ANDLER à Emmanuel LEVY, L'Affirmation du droit collectif. Conférence faite à Lyon le 17 mars 1903, Paris, Société nouvelle de librairie et d'édition, 1903, p. 3.

48. Préf. de C. Andler à Anton Menger, Le Droit au produit intégral du travail. Etude historique, trad. Alfred BONNET, Paris, Giard et Brière, 1900, p. XXXIV.

49. Ibid., p. XXXIV.

50. Ibid., p. XX. 
Andler, en recourant à la dimension juridique du socialisme, prétendait renouer avec l'ancienne tradition socialiste française. Marx s'était fourvoyé, perdu dans son économisme. Une des caractéristiques majeures du socialisme de Charles Andler est de faire effort pour échapper au poids de l'économie, pour mettre en avant des exigences morales et juridiques. Le marxisme, selon lui, n'avait pas su trouver cette voie :

« [...] on peut dire que le marxisme, à force de s'adonner, avec une exactitude minutieuse, mais absorbante, à la critique des formes économiques de la production, a négligé l'étude et la critique des formes transitoires du droit. On s'est ingénié sans doute à trouver dans le marxisme lui-même un contenu juridique; et ce contenu n'est pas niable, mais la peine qu'il faut se donner pour le découvrir en prouve la pauvreté ${ }^{51}$.

Ce droit nouveau germait déjà dans la société française. Les progrès de l'organisation syndicale et coopérative auxquels ne pouvait manquer d'être sensible un militant du parti allemaniste, méfiant à l'endroit du parlementarisme, le révélaient. Plusieurs marxistes « dissidents » de la fin du siècle, tout en remettant en cause les données traditionnelles de l'économie marxiste proposaient de nouveaux modes d'intervention au mouvement ouvrier. Un mouvement intellectuel - celui de "révision » du marxisme - s'épanouissait dans un mouvement politique. Andler, tout en se posant en historien érudit, n'ignorait rien des nécessités politiques. Comme Bernstein mais aussi Kampffmeyer ou Adèle Gerhard, comme les socialistes belges et les Fabiens anglais, il concluait de sa propre critique de l'économie marxiste que l'émancipation de la classe ouvrière passait par la constitution de coopératives de consommation.

Fidèle aux analyses d'Otto Effertz, Andler contestait radicalement la théorie de la valeur-travail. Il existait selon lui une partie de la valeur d'une marchandise que le travail ne produisait pas. Cette partie de la valeur provenait du sol. La matière première brute avait une valeur que Marx aurait eu tort de négliger. Andler reviendra sans cesse sur cette «faille » qui paraissait, évidemment, renverser tout le système de Marx $^{52}$ mais aussi qui, par le même coup, fondait une «justice boiteuse " :

" Une justice qui ne se préoccupe que de l'échange des quantités égales de travail est boiteuse. Il faut qu'elle se complète par une tarification des matières premières qu'ensuite on répartira, proportionnellement au travail fourni par chacun. Toute espèce de rente attachée au sol et à la propriété des

51. Préf. de C. ANDLer à E. Levy, op. cit. supra n. 47, p. 5. C'est à l'évidence Sorel qui était ici visé.

52. Préf. de C. ANdler à A. Menger, op. cit. supra n. 48, p. XXXIV. 
matières premières aura ainsi disparu. Une quantité définie des produits du sol consommables sera affectée à tout homme en échange d'un travail dont il faudra convenir. Mais il y aura une rente attachée à la qualité du labeur. L'organisation syndicale qui survivra aux luttes actuelles, garantira aux travailleurs un supplément de rémunération attaché à l'habileté [rent of ability] ou à l'utilité urgente de leur effort [rent of opportunity] " ${ }^{53}$.

A un second niveau d'analyse, Andler reprochait à Marx d'avoir concentré ses efforts sur la production et négligé la consommation et l'échange. Or, écrivait Andler, « contrairement à ce que pensait Marx, la formation des plus-values a lieu dans l'échange. La vraie exploitation se passe dans l'entremise entre le producteur et le consommateur $\gg{ }^{54}$. Pour le reste, Marx avait eu raison : rapines, pillages, vols dépossédaient bien une classe de travailleurs au profit d'une autre classe, non celle des détenteurs de moyens de production mais celle des « marchands déprédateurs ${ }^{55}$. Le capital n'était pas par essence exploiteur et ce n'était que par la vente que la plus-value se réalisait.

De ce constat, Andler tirait les conséquences en militant socialiste. C'est vers la consommation, en jouant de sa formidable force d'achat, que la classe ouvrière devait orienter son action. D'où le rôle éminent que devaient remplir les coopératives de consommation dans l'avènement de la République sociale. Peu à peu, en se fédérant les unes aux autres, en annexant des ateliers productifs, les coopératives de consommation accompliraient la révolution sociale, pacifiquement, sans générer de phénomène bureaucratique et dans la liberté.

Toutes ces analyses avaient donné à Andler une inébranlable réputation d'adversaire de Marx. Sa critique, il est vrai, n'avait pas été tendre et malgré quelques tentatives parfois pour épargner Marx afin de mieux égratigner les marxistes, les glissements de l'un à l'autre contribuaient à confondre les deux. Il n'est pas certain qu'Andler ait toujours pratiqué cette confusion avec conscience. L'introduction historique et le commentaire - ce sont là ses propres termes - adjoints à la nouvelle traduction qu'il fit du Manifeste communiste le montrent bien. Les « marxistes orthodoxes ", en France comme en Allemagne, crièrent au scandale. Ayant, dans un premier temps, rendu hommage au travail d'Andler, Mehring en vint rapidement à une polémique assez vive. Il avait certes d'abord reconnu « un travail sérieux, approfondi et qui fait honneur à l'écrivain " et avait même ajouté qu'avec "des adversaires comme lui [Andler], les problèmes du marxisme peuvent se discuter sérieuse-

53. Ibid., p. XXXIV.

54. C. ANDLER, " Le rôle social des coopératives », Revue de métaphysique et de morale, janv. 1900 , p. 127.

55. Ibid., p. 128. 
ment $"{ }^{56}$. Mais il avait aussitôt remis en cause les résultats auxquels était parvenu Andler, lui reprochant de "considérer Marx comme une "colonne Morris", sur laquelle je collerais toutes les citations qu'on peut tirer sans peine de tel ou tel socialiste français ${ }^{57}$. Au demeurant, Mehring ne s'était peut-être pas trompé en percevant derrière la démarche d'Andler une volonté de remettre directement en cause le marxisme de la social-démocratie. L'âpreté de l'échange semble en tout cas indiquer qu'il ne s'agissait pas là simplement d'une divergence d'interprétation opposant deux savants mais bien plutôt d'une querelle d'essence toute politique : ne vit-on pas l'atrabilaire Andler évoqué sous les traits d'un "noble Peau-Rouge" sortant de "quelque baraque foraine", ou ceux de "reptile" dont il fallait "montrer encore les crochets venimeux particuliers à son espèce " ${ }^{58}$ ? Gageons que la sympathie, déjà fort modérée, qu'Andler éprouvait pour la social-démocratie allemande n'en fut pas augmentée.

A la base de cette polémique, il y avait donc le Commentaire du Manifeste communiste que Charles Andler avait publié en 1901. Sa thèse, il le reconnaissait lui-même, n'était pas entièrement neuve. Il prétendait seulement, au moyen d'un commentaire historique serré, mettre en valeur les influences qu'avaient subies Marx et Engels. Il y découvrait, pour le meilleur et pour le pire, plusieurs traditions socialistes enchevêtrées. La lutte des classes comme moteur de l'histoire était déjà présente chez Babeuf mais aussi chez Blanqui et Bazard ${ }^{59}$. L'ascension historique de la bourgeoisie par les progrès de l'industrialisme devait beaucoup à Proudhon, à List, à Pecqueur, à Vidal, à Sismondi et à Buret ${ }^{60}$. Pecqueur en France, List en Allemagne avaient, les premiers, édifié " un système complet de matérialisme historique " ${ }^{61}$. Marx avait emprunté à Pecqueur la description des nouvelles valeurs de la bourgeoisie. Engels avait construit sa théorie des crises de surproduction en «bon disciple de Sismondi ${ }^{62}$. Et Andler multipliait ainsi les exemples mettant surtout en avant les emprunts que Marx et Engels avaient faits à la tradition française du socialisme.

Dès lors, que restait-il de propre aux deux auteurs allemands? Para-

56. Franz MeHrING, « Le manifeste communiste », Le Mouvement socialiste, 8 févr. 1902, p. 249.

57. ID., "Réponse à Charles Andler ", Le Mouvement socialiste, 5 avr. 1902, p. 634.

58. Ibid., p. 642 et 643.

59. Karl MARX, Friedrich EnGels, Le Manifeste communiste. Introduction historique et commentaire par C. ANDLER, Paris, Société nouvelle de librairie et d'édition, 1901, p. 65 .

60. Ibid., p. 71

61. Ibid., p. 73.

62. Ibid., p. 99. 
doxalement, le Commentaire du Manifeste est le texte le moins virulent de la période. Andler y reconnaît les grands mérites de Marx : la justesse de certaines de ses analyses historiques ou la qualité de sa sociologie, en particulier un " regard perçant » sur les attitudes du Lumpenproletariat et des classes moyennes ${ }^{63}$. Mieux encore, le texte s'achève sur une appréciation élogieuse du matérialisme historique :

" D'emblée il a exclu le dogmatisme. Il ne peut s'enfermer en des formules immobiles. Il absorbe en lui la teneur de toutes les théories et le profit de toutes les expériences. II institue, comme l'a dit Engels dans son article sur Carlyle (1844), “ un mouvement de pensée qui ne se lie à aucun résultat fixe, mais qui dépasse incessamment les résultats acquis; une pratique qui ne s'attache à aucune position acquise, mais dépasse ces positions antérieures".

En ce sens on peut dire de lui qu'il fonde la méthode révolutionnaire éternelle ${ }^{64}$.

Par cette édition commentée du Manifeste, les marxistes étaient plus menacés que Marx. Le texte était désacralisé, devenu objet d'histoire. Pire que mort, Marx avait vécu. Andler acquit ainsi définitivement une réputation d'antimarxiste aux yeux des marxistes. Cette édition qui devint une référence pour tous ceux qui s'intéressaient à Marx le coupa à jamais du « marxisme orthodoxe ».

\section{L'ANTIMARXISME INTROUVABLE?}

A partir de 1903, Andler se consacra moins à Marx et atténua sa critique. Après 1906, il renonça même tout à fait à écrire de nouveaux textes critiques. Son étude sur Frédéric Engels, qui parut fin 1913-début 1914 , avait été rédigée vers 1897 , lorsqu'il envisageait de publier sa Décomposition du marxisme. La publication tardive de ces articles était, en outre, conjoncturelle et faite "pour répondre à des reproches récents qui incriminaient [son] "scepticisme socialiste déjà ancien", croyait-on, ou [sa] "vieille haine du marxisme" " ${ }^{65}$. Charles Andler, après la polémique qui l'avait opposé à Jaurès, se trouvait alors de plus en plus marginalisé au sein du Parti socialiste.

Durant des années, nombreux furent les intellectuels qui s'éloignèrent

63. Ibid., p. 127.

64. Ibid., p. 208-209.

65. C. ANDLER, "Frédéric Engels. Fragment d'une étude sur la Décomposition du marxisme ", La Revue socialiste, nov. 1913, p. 385. 
de leurs préoccupations socialistes. Andler suivit ce mouvement. Encore faut-il remarquer qu'il adhéra à la S.F.I.O. en 1905 et qu'il y accomplit même un travail de militantisme intellectuel important notamment lors de la fondation de l'École socialiste ${ }^{66}$. En ce sens, il fut plus engagé dans la vie politique que la plupart des intellectuels socialistes de cette période post-dreyfusienne.

Il poursuivit parallèlement son travail de critique du marxisme mais de façon de plus en plus docte et de moins en moins polémique. Les hommages rendus à Marx se font plus fréquents ${ }^{67} \mathrm{et}$, à plusieurs reprises, il voulut se défaire de son image d'antimarxiste invétéré. Il se présenta en érudit, non en polémiste. Mais Andler était aussi un militant politique, adversaire du guesdisme, autrement dit du marxisme politiquement incarné.

Trois textes importants lui permirent de revenir sur les thèmes de sa critique de Marx. Il les développait à présent avec sérénité, renonçant à la thématique du "pillage", érudite, certes, mais aussi polémique. On pourrait même dire qu'une bonne partie du travail d'Andler avait dorénavant pour but de couper Marx des marxistes, « hétérodoxes " compris. Il s'en prit ainsi à la théorie des mythes sociaux au moyen de laquelle il pensait que Sorel avait voulu sauver Marx de ses erreurs. Or, selon Andler, Sorel se trompait tout à fait sur la pensée de Marx :

"A l'époque où est né le socialisme qui s'est dit scientifique, on ne croyait pas "être voisin de la science" en exposant sa doctrine sous la forme d'un mythe; et l'on ne sauvera pas le catastrophisme de Marx, opinion sincère de son fanatisme sardonique, par cette interprétation désespérée ${ }^{68}$.

De même, contre Menger, il pense que le marxisme ne peut se réduire à une interprétation économique de l'histoire puisque Marx a reconnu le rôle de la force, même s'il n'a pas su se faire une idée de son importance ${ }^{69}$. Il s'oppose aussi à Menger pour affirmer avec Marx que les révolutions ne peuvent se réduire à des manigances de coterie. Il y a derrière des forces sociales, et Andler reprend à son compte l'ensemble

66. Cf. C. Prochasson, « Jaurès, l'École socialiste et le socialisme normalien », Bulletin de la Société d'études jaurésiennes, 84, janv.-mars 1982, p. 3-7.

67. Cf. préf. de C. ANDLER à Albert ThOMAs, Le Second Empire (1852-1870), Paris, Rouff, s.d.

68. Préf. de C. ANDler à Ferdinand LASSALLe, Théorie systématique des droits acquis. Conciliation du droit positif et de la philosophie du droit, trad. franç. d'après la $2^{e}$ éd. allemande par J. Bernard, J. Molmor, G. Mourllet, A. Weill, Paris, Giard et Brière, 1904 , t. 1, p. XVII.

69. Introd. de C. ANDLer à Anton Menger, L'État socialiste, trad. d'Edgard MilHaud, Paris, Société nouvelle de librairie et d'édition, 1904, p. VI. 
de l'analyse que Marx fait du coup d'État de Louis-Napoléon Bonaparte ${ }^{70}$. Andler ira même jusqu'à admettre le poids déterminant de l'économique dans l'histoire, particulièrement en matière juridique, idée qu'il avait pourtant contestée auparavant. Il affirma enfin, d'accord avec Marx, "que le degré de civilisation des peuples dépend des conditions de leur production économique $"{ }^{71}$.

Il maintint ferme, en revanche, son opposition à la théorie de la valeur de Marx, proposant sa propre théorie, nourrie des travaux d'Otto Effertz :

"La théorie marxiste échangerait un bon livre contre plusieurs chevaux, puisque ces produits différents ont peut-être coûté la même somme de travail, surtout en tenant compte de ce que la production d'un livre est du travail qualifié. Mais l'invraisemblance éclate. C'est que Marx n'a pas considéré la quantité de terre incorporée dans la valeur. Il n'a pas la notion du rapport travail-terre $\left(\frac{\mathrm{a}}{\mathrm{b}}\right)$. C'est ce rapport seul qui rend les objets comparables entre eux ${ }^{72}$.

Ce désaccord avec Marx est évidemment fondamental. Il n'empêche qu'Andler ne parlait plus alors ni de ruine ni même de décomposition du marxisme. Il allait même jusqu'à en retenir des pans importants. Autrement dit, il y avait du bon et du mauvais Marx. Et le mauvais Marx provenait souvent des interprétations, voire des falsifications, que son ami Engels avait produites.

La dissociation du couple Marx-Engels était un thème récurrent chez les révisionnistes. La pensée pure de Marx, selon eux, avait été victime de sa vulgarisation. Elle s'était dégradée en politique. Dès 1892, Andler avait, précocement, lui aussi, opéré cette distinction ${ }^{73}$. Tout en faisant, ici et là, de sérieux reproches à Engels (sa responsabilité dans le gel politique du marxisme, son attitude et ses erreurs au cours de la polémique avec Dühring, etc.), il n'avait pas hésité à saluer certains de ses travaux. Dans le Commentaire du Manifeste communiste, il avait évoqué les " résultats lumineux et simples " qui émanaient de la description du sort de la classe ouvrière dans les Classes laborieuses en Angleterre ${ }^{74}$.

Dans la longue étude qu'Andler consacra à Engels dans la Revue socialiste, on retrouve dans les critiques pareil balancement. Si «le

70. Ibid., p. XVII.

71. C. ANDLER, art. cit. supra n. 33, p. 602. Cet article reprend à peu près exactement (à l'exception de l'introduction et de quelques très légères modifications stylistiques) l'introduction qu'Andler donna aux Antagonismes économiques d'Otto Effertz.

72. Ibid., p. 600 .

73. Cf. art. cit. supra n. 25 , p. 246.

74. Cf. op. cit. supra n. 59, p. 113. 
Capital a limité les esprits " ${ }^{75}$, la faute en revenait plus aux successeurs de Marx qui ont cru trouver un marxisme (en réalite il y a des marxismes, souligne Andler) en l'état d'une dogmatique toute faite, prête à penser. La complexité du texte de Marx ne pouvait en outre faciliter les interprétations d'autant plus que les « disciples " avaient contribué à brouiller les pistes : ils eurent le tort, selon Andler, d'essayer de

« compléter la doctrine quand Marx l'avait sciemment laissée inachevée. Mais surtout ils l'ont reconstruite et modifiée à leur insu, quand ils ne croyaient que la reproduire. Ces vieux disciples les plus fidèles dans leur intention, et qui avaient encore connu Marx, sont justement ceux qui l'ont le moins compris $"{ }^{76}$.

Engels fit partie de ceux-ci, lui, dont l' « influence destructrice " avait "vigoureusement contribué à décomposer le marxisme ": "Avoir survécu quinze ans à la mort de Marx, c'est avoir couru beaucoup de chances de changer d'idées $"{ }^{77}$.

La critique fut vive : trahison, confusion, emploi abusif d'un langage métaphorique, métaphysique grossière, pauvreté de la sociologie, rousseauisme dans la théorie de la famille, artifice de la notion de " dernière instance". Mais si Engels n'avait pas été un grand savant, il avait en revanche été un grand socialiste. Et pour Andler cet hommage-là avait son prix :

" De tous les livres qu'a produits le marxisme, ceux de Frédéric Engels sont assurément les plus compréhensifs, et les seuls qui fassent apparaître, avec les principes moraux, sociologiques et logiques du système, les conséquences pratiques où il tend. L'unité forte de la pensée est un mérite que Marx luimême n'offre pas à ce degré. Il convient d'ajouter maintenant que cette unité est imaginative plutôt que scientifique. Personne plus qu'Engels n'a converti d'hommes au socialisme; et personne n'a plus aventuré la doctrine. Si le marxisme n'était que ce qu'il paraît chez Engels, il y a longtemps qu'il ne compterait plus dans la science $"{ }^{78}$.

75. C. ANDLER, art. cit. supra n. 65. L'article a été publié sur 4 numéros : nov. 1913 (p. 385-397), déc. 1913 (p. 481-501), janv. 1914 (p. 54-76), févr. 1914 (p. 147-168). Andler assura n'avoir pas retouché son article. Il en a au moins actualisé la bibliographie puisque est cité le livre de Rodolfo MONDOLFO, Il Materialismo storico in Federico Engels publié en 1912. Nous pensons que, contrairement à ses dires, Andler a remanié son texte. C'est la raison pour laquelle nous le plaçons dans le deuxième temps de sa critique.

76. Ibid., nov. 1913 , p. 386 .

77. Ibid., p. 387.

78. Ibid., janv. 1914 , p. 70. 
Si Andler avait, un temps, attaqué Marx et Engels de façon si agressive, n'est-ce pas sans doute qu'il vivait mal une ambiguïté caractéristique du marxisme, celle qui mêle la démarche scientifique ou savante à l'engagement social et politique? Le socialisme andlérien était trop éthique pour pouvoir être marxiste. Cela ne signifie pas qu'Andler ait été un adversaire de la pensée de Marx, comme d'autres socialistes. Ce qu'il excluait était la revendication de légitimité scientifique du marxisme au sein d'un combat social. Ce mélange des genres lui était insupportable.

On pourra, en outre, faire remarquer que les attaches socialistes de Charles Andler n'étaient pas susceptibles de le sensibiliser à la politique marxiste, en tout cas à celle de la social-démocratie allemande ou, en France, à celle des guesdistes. La base de son engagement politique est morale. Comme Sorel, mais en un sens différent, plus métaphysique, Andler parle d'une « moralité des producteurs » et d'une nouvelle moralité socialiste ${ }^{79}$. De plus en plus nourri d'un individualisme nietzschéen, il s'éloigne d'une conception qui identifie le socialisme à un nouveau mode de production. Si la classe ouvrière est une classe d'avenir, c'est avant tout parce que la bourgeoisie est une classe en décadence morale, qui a usé tout son capital d'idéal. Une critique respectueuse de la démocratie (qui a accompli son œuvre historique) accompagne des thèses andlériennes qu'on aurait tort d'interpréter comme annonciatrices du seul néo-socialisme de l'entre-deux-guerres.

Le projet politique d'Andler est tout autre. Sa volonté de retourner aux théoriciens du socialisme français n'est pas seulement animée d'une sensibilité nationale hostile à la pensée allemande (car la pensée allemande est aussi un objet de fascination). Certes, Andler a plus de sympathie pour Proudhon que pour Marx. Il alla jusqu'à regretter qu'un système allemand à l'élaboration duquel la classe ouvrière française n'avait pris aucune part soit parvenu, du fait de circonstances historiques particulières, à éliminer les doctrines du socialisme libertaire et contractuel. Mais Andler n'ignorait pas non plus les faiblesses de Proudhon, victime comme Marx, de ses partisans : "[...] le proudhonisme des disciples s'est borné à la répétition stérile de formules vieillies ${ }^{80}$.

Il fallait, malgré cela, revenir à Proudhon. Il fallait reconstituer une armature théorique au socialisme de tradition française. Non pour s'opposer à Marx mais pour faire reculer l'hégémonie que les marxistes

79. C. ANDLER, La Civilisation socialiste. Sténographie d'une leçon de clôture prononcée à l'École socialiste le 3 juin 1910, Paris, Marcel Rivière, Les documents du socialisme, 1911 .

80. ID., préf. à Jules L. PUECH, Le Proudhonisme dans l'Association internationale des travailleurs, Paris, Alcan, 1907, p. XVI. 
avaient acquise dans le socialisme français. Pour Andler, leur présence constituait un blocage politique. Leurs analyses les cantonnaient dans une attitude d'attente qui faisait obstacle à l'avènement de la République sociale.

Dès 1897, Andler avait songé à fédérer tous les éléments progressistes de la vie politique française pour instituer un "socialisme d'État " :

« J'appelle socialisme d'État une théorie qui pense que les choses sociales sont affaire de gouvernement [...], tandis qu'il y a des doctrines qui rejettent toute règle sociale ou qui croient (comme un certain pseudo-marxisme) que tous les changements sociaux se font d'eux-mêmes " ${ }^{81}$.

L'inspiration fabienne est claire. Andler percevait bien l'existence d'un « socialisme libéral " qui unirait des socialistes comme Georges Renard pour lequel il eut toujours une grande admiration (n'était-il pas un héritier direct du socialisme quarante-huitard?), mais aussi des disciples de Walras, des radicaux et tous les socialistes non marxistes ${ }^{82}$.

Les positions de Charles Andler face au marxisme ne pourraient pas se comprendre sans ces éléments d'analyse politique. Elles ne pourraient pas non plus se comprendre si l'on ne faisait pas référence au profond sentiment national qui troublait les rapports qu'entretenaient alors tous les intellectuels français avec l'ensemble de la culture allemande ${ }^{83}$.

\section{MARX EST ALLEMAND}

On a plusieurs fois souligné le handicap que l'origine allemande de la pensée marxiste constituait pour sa diffusion en France. Au tournant du siècle, les intellectuels français ne cultivaient pas des rapports simples avec la culture allemande. Attirés, fascinés, inquiets, hautains, ils sont tous pris dans un complexe national ravivé dans les années qui suivirent Tanger.

Chez Andler, le phénomène se complique. Alsacien jusqu'en 1879, il était arrivé en France maîtrisant mal le français, gêné dans la poursuite de sa scolarité. Normalien, il échoua à l'agrégation de philosophie et fut

81. Lettre de C. Andler à G. Renard du 2 août 1897, Fonds Georges Renard, t. 96, MS 2577, Paris, B.H.V.P.

82. Ibid.

83. Sur ce sujet, cf. Claude Digeon, La Crise allemande de la pensée francaise, Paris, P.U.F., 1959. 
contraint, la mort dans l'âme, de passer celle d'allemand. Et le voici devenu à tout jamais le grand germaniste français, Charles Andler... L'Allemagne, la culture allemande furent désormais au coeur de sa vie, non sans fracas : en mai 1908, il dut subir les insultes des journaux nationalistes (l'Autorité attaquait le «cuistre Andler » et l'Éclair reprochait à ce " théoricien notable du socialisme " d'avoir accompagné des étudiants en Allemagne); en 1913, une grave polémique sur la socialdémocratie allemande l'opposa à Jaurès. L'Allemagne, toujours l'Allemagne... La guerre le vit, enfin, se livrer à des exercices littéraires d'un patriotisme échevelé. Il s'opposa violemment à tout courant pacifiste, condamna Zimmerwald et Kienthal, et adhéra, fin mai 1917, à la protestation des quarante députés socialistes contre la conférence de Stockholm.

Andler ne fut pas le seul intellectuel français à déployer une germanophobie outrée durant la guerre. Nombreux furent ceux qui adoptèrent semblable attitude au mépris de leur réserve antérieure. Le socialisme allemand, associé aux caractères de l'Allemagne tout entière, dut subir le harcèlement critique de Charles Andler : «[...] le socialisme allemand souffre du grand mal dont souffre l'Allemagne actuelle: le manque d'originalité, l'arrêt intellectuel ${ }^{84}$. L'œuvre de Marx elle-même est marquée, aux yeux d'Andler, du sceau de la germanité et il n'aura de cesse d'opposer le socialisme allemand au socialisme français. Proudhon contre Marx, c'est aussi garance contre casque à pointe : « Nous ne croyons pas à cette différence de tempérament. Nous croyons à des différences de culture ${ }^{85}$. Pas de psychologie donc, mais de l'histoire. C'est dans cette optique qu'il faut comprendre la série de conférences consacrées à la philosophie allemande au XIX ${ }^{e}$ siècle qu'il organisa, aidé par Dominique Parodi et Lucien Herr, dans le cadre de l'École des hautes études sociales ${ }^{86}$. On ne pourra rendre compte de cette volonté de connaître la culture allemande par des motivations du seul ordre scientifique. Il ne s'agit pas seulement de montrer ce qu'il y a de français dans Marx ou d'affirmer que "de Feuerbach est sorti Marx ${ }^{87}$ au profit de l'enrichissement de la connaissance humaine. Il y a là aussi un défi national : préparant la commémoration du centenaire de Schiller, Andler ne manqua pas de faire remarquer que les Français attendraient

84. L. GERSAL, op. cit. supra n. 24, p. 245.

85. Préf. de C. ANdler à A. Menger, op. cit. supra n. 69, p. XVIII.

86. C. ANDLER, préf, à C. ANDLER et al., La Philosophie allemande au XIX siècle, Paris, Alcan, 1912.

87. ID., préf. à F. LASSALLE, op. cit. supra n. 68, p. XIII. 
" longtemps que les Allemands fassent une manifestation analogue sur le nom d'un Français. Ils n'ont pas su le faire sur le nom de Descartes. Nous voulons montrer que nous valons mieux qu'eux, par la compréhension de la pensée des autres nations ${ }^{88}$.

Andler, avec la guerre, passa de la décomposition du marxisme à la Décomposition politique du socialisme allemand. Il s'agit des deux faces d'une même préoccupation : décomposer l'Allemagne, en révéler la barbarie. L'Allemagne n'aime pas la démocratie, le socialisme allemand la méprise et n'eut toujours que dédain pour l'ordre républicain. Le socialisme allemand était du même ordre que le militarisme allemand. Sûr de lui, hautain, enlisé dans un esprit gestionnaire et bureaucratique, les socialistes allemands avaient renoncé à tout idéal révolutionnaire ${ }^{89}$.

L'hégémonie du marxisme était la clef de cette évolution. On trouve chez Marx, selon Andler, le même mépris de la démocratie que chez les socialistes allemands. La barbarie marxiste avait éliminé du socialisme allemand toute idée de droit et, l'esprit socialiste, lorsque la guerre éclata,

" était éteint depuis longtemps, étouffé par la haine marxiste de la justice, de la liberté, en un mot de l'idéologie. Les derniers livres de socialisme allemand où il est question du droit sont ceux de Lassalle. Le marxisme a enseigné pendant deux générations la seule étude monographique des faits économiques, et la seule recherche des moyens qui permettront d'en tirer le plus fort rendement pour le prolétariat. Ce serait une erreur de croire que dans cette étude et dans cet effort d'organisation purement matériel, on rencontre nécessairement l'idée de droit et de liberté. Quand les intérêts égoïstes de la classe ouvrière allemande se sont trouvés solidaires d'une action conquérante et d'un régime de force, c'est donc la conquête et la force que la social-démocratie a préférées, au nom des intérêts strictement allemands $"$ 90 .

La guerre venait donc révéler les négligences de Marx en matière juridique qu'Andler avait déjà signalées avant-guerre ${ }^{91}$. Cette thématique du droit venait aussi s'accorder avec les besoins du « bourrage de crâne " : les Français ne faisaient-ils pas la guerre du droit ? Ce fut dans cette optique, harmonisant ses anciennes préoccupations théoriques avec

88. Letțre de C. Andler à Paul Léon, s.d. (mars 1905 ?), communiquée par M. Jean-Paul Léon.

89. Cf. C. ANDLER, La Décomposition politique du socialisme allemand 1914-1919, Paris, Bossard, 1919. Il s'agit d'un recueil d'articles publiés dans L'Action nationale de nov. 1917 à févr. 1919.

90. Ibid., p. 86.

91. Cf. supra, p. 95. 
les nouveaux besoins d'une propagande nationale, qu'Andler relut Marx et Engels. Il trouva chez eux tous les traits de la sauvagerie teutonne : l'assurance aveugle, le militarisme («[...] Engels n'a pas cessé de préconiser le système [militaire] prussien. Il y avait là une de ces vieilles préférences nationales, indéracinables chez un bon Prussien $"{ }^{92}$ ), la grossièreté, le nationalisme ( Marx et Engels, en 1870, souhaitaient puissamment la victoire allemande ${ }^{93}$ ), la haine ( $($ marxiste $»)$. L'interprétation andlérienne du marxisme s'était-elle alors dégradée en politique?

Pas tout à fait. Andler ne fit alors que reprendre ses analyses antérieures, certes, avec plus de vigueur. La guerre était d'évidence un moment privilégié pour abattre l'édifice théorique du marxisme. Le système de Marx était cette fois-ci devenu périmé ${ }^{94}$. Son erreur essentielle, dévoilée par la guerre, est le primat qu'il accordait à l'économique. Or, aux yeux d'Andler, on ne peut faire l'impasse ni sur l'idée démocratique, ni sur l'idée républicaine : «[...] tout, en effet, est affaire de "suffrage" et de "forme politique" " ${ }^{95}$. De l'économisme de Marx, les marxistes avaient tiré, sans nuance, une politique d'abord inadaptée puis bientôt pleine des plus grands dangers pour la démocratie. Il était faux de dire que l'Empire équivalait à la République, quand bien même les ouvriers allemands jouissaient de lois sociales plus avancées que celles dont disposaient les ouvriers français.

La révolution russe de février 1917 ne souleva pas l'enthousiasme d'Andler. En mai, il écrivit à Marie Allart : « [...] cela marche mal. Voilà un grand crime à l'actif des marxistes russes, germanophiles ${ }^{96}$. Trois mois plus tard, alors que la situation économique et sociale russe se détériorait, Andler se mit à faire de la théorie le moteur de l'histoire :

« [...] passé deux heures de l'après-midi à Pétrograd, on ne peut même plus acheter un timbre-poste, parce que tout le monde estime qu'à deux heures, la journée de travail est terminée. Ceci est du bon marxisme. Vous vous rappelez que dans le Capital le calcul de la plus-value se fait toujours à partir d'une journée de travail de 6 heures, nécessaire à produire la subsistance de l'ouvrier. Admirable effet des déductions algébriques qui partent d'une hypothèse numériquement arbitraire $"{ }^{97}$.

92. C. ANDLER, op. cit. supra n. 89, p. 42.

93. Ibid., p. 110.

94. C. ANDLER, Le Socialisme impérialiste dans l'Allemagne contemporaine, Paris, 1918.

95. ID., op. cit. supra n. 89, p. 280.

96. Lettre de C. Andler à M. Allart du 13 mai 1917, I.F.H.S., 14 AS 188.

97. Lettre de C. Andler à M. Allart du 16 août 1917, I.F.H.S., 14 AS 188. 
La Révolution d'octobre lui permit, enfin, de faire couler des flots d'ironie. Qu'allaient penser les marxistes d'une révolution qui ne respectait ni les étapes ni les lois dialectiques qui liaient infrastructure et superstructure? D'où, selon lui, l'attitude des socialistes allemands : "Une catastrophe qui se moque de la dialectique n'est pas une catastrophe marxiste $"$ 98 .

Le socialisme marxiste allemand, ainsi que Charles Andler l'avait annoncé avant-guerre, semblait avoir failli. La brutalité politique d'un Ebert ou d'un Noske achevait de le discréditer. Kautsky, ce pape du marxisme, s'était trompé et avait "contribué à l'abrutissement où se débat le socialisme allemand d'aujourd'hui " ${ }^{99}$. Quant au spartackisme, il n'était rien d'autre "que la forme outrée d'un certain chauvinisme exaspéré et pessimiste, qui aime mieux abîmer l'Allemagne entière dans l'incendie d'une révolution universelle que de la déclarer seule vaincue " ${ }^{100}$. Certes Andler rendait hommage à son internationalisme. Mais était-il sincèrement autre chose qu' " une astuce de guerre allemande " ${ }^{101}$ ? On pouvait, selon lui, en douter. Seul Kurt Eisner sauvait le socialisme allemand car lui seul s'était « trouvé du côté de Jaurès; et il n'a pas eu tort de penser qu'il manquait beaucoup à une démocratie socialiste, qui n'était pas d'abord une démocratie politique " ${ }^{102}$.

On pourra clore ici l'examen de la critique andlérienne du marxisme. Dans l'entre-deux-guerres, Andler ne se consacra plus guère qu'à ses études nietzschéennes. Le conflit mondial, puis les révolutions russes de 1917 l'avaient éloigné de toute tentation marxiste. Il serait cependant inexact de ne retenir que la dernière période au cours de laquelle Andler confondit avec application l'œuvre de Marx et le "marxisme orthodoxe " de la social-démocratie allemande. Ce qu'il avait pourtant longtemps essayé de distinguer par souci d'exactitude, il le mêlait de nouveau par affection nationale. Andler avait toujours discuté Marx, voire radicalement contesté dans ses fondements les plus essentiels. Mais il ne l'avait jamais réduit à l'état d'une pensée politique néfaste, à l'envergure médiocre et aux ambitions méprisables. Il le fit, pourtant, dans ses articles et ouvrages écrits durant la guerre, tous aspirés par l'idéologie nationale qui redonnait toute sa valeur à un socialisme français, libéral, modéré, et non marxiste. La guerre fut une chance pour cette pensée-là.

98. C. ANDLER, op. cit. supra n. 89, p. 164.

99. lbid., p. 152 , n. 1 .

100. Ibid., p. 229. Rosa Luxembourg est qualifiée de «marxiste polonaise au service du socialisme allemand ", "dialecticienne retorse » et Andler ajoute qu'elle est "une des plus agissantes théoriciennes du marxisme outrancier » (p. 102).

101. Ibid., p. 240.

102. Ibid., p. 279. 
Du cas Andler, pourra-t-on tirer une conclusion profitable à l'histoire intellectuelle du socialisme? De cet indéniable fait - le marxisme s'est aussi diffusé par des canaux non marxistes ${ }^{103}$ - pourra-t-on mettre en valeur l'existence d'une réception dont les historiens des idées n'auraient peut-être pas encore suffisamment souligné l'importance? On pourra en tout cas se poser plusieurs questions : que signifie être partisan d'une doctrine? Comment s'en opère la diffusion? Les caractéristiques de l'œuvre de Marx, il est vrai, rendent les réponses à ces questions encore plus délicates. Des enjeux politiques viennent y troubler toute approche scientifique. Charles Andler, lui-même à la fois socialiste et savant, se heurta au double statut du marxisme et ne parvint que difficilement à faire la part des choses. L'acharnement qu'il mit à détruire la légitimité philosophique et scientifique du marxisme le révèle. Qu'il le fasse dépendre d'une tradition française antérieure ou qu'il lui cherche des substituts (Effertz, Landry, etc.), Andler réduisit toujours la pensée de Marx à un objet historique mort.

Il faudrait, en tout état de cause, distinguer la connaissance du marxisme de sa diffusion. On aurait tort, cependant, d'opposer ces deux aspects. L'exemple de Charles Andler le montre bien. Non marxiste, sinon antimarxiste, remarquable connaisseur de l'œuvre de Marx - nous ne prétendons pas ici discuter de la valeur de cette connaissance - il s'en fit aussi le médiateur : il la discute en différents lieux, il en rend compte, quand il ne la traduit pas... Un mode singulier de diffusion pourrait bien exister pour le marxisme comme pour d'autres théories que le terme de " réception " évoquerait volontiers.

Christophe Prochasson, Lycée Michelet, Vanves.

103. Rappelons qu'Elie Halévy était un remarquable connaisseur de l'ceuvre de Marx dès la fin des années 1890 . 